CLINICAL STUDY

\title{
Post-surgical thyroid ablation with low or high radioiodine activities results in similar outcomes in intermediate risk differentiated thyroid cancer patients
}

\author{
Maria Grazia Castagna ${ }^{1}$, Gabriele Cevenini ${ }^{2}$, Alexandra Theodoropoulou ${ }^{1}$, Fabio Maino ${ }^{1}$, Silvia Memmo ${ }^{1}$, \\ Cipri Claudia $^{1}$, Valentina Belardini ${ }^{1}$, Ernesto Brianzoni ${ }^{3}$ and Furio Pacini ${ }^{1}$ \\ ${ }^{1}$ Section of Endocrinology and Metabolism, Department of Internal Medicine, Endocrinology and Metabolism and Biochemistry and ${ }^{2}$ Department of \\ Surgery and Bioengineering, University of Siena, Policlinico Santa Maria alle Scotte, Viale Bracci 1, 53100 Siena, Italy and ${ }^{3}$ Unit of Nuclear Medicine, \\ Macerata Hospital, 62100 Macerata, Italy \\ (Correspondence should be addressed to F Pacini; Email: pacini8@unisi.it)
}

\begin{abstract}
Background: In differentiated thyroid cancer (DTC) patients at intermediate risk of recurrences, no evidences are provided regarding the optimal radioactive iodine (RAI) activity to be administered for post-surgical thyroid ablation.

Methods: This study aimed to evaluate the impact of RAI activities on the outcome of 225 DTC patients classified as intermediate risk, treated with low (1110-1850 MBq) or high RAI activities $(\geq 3700 \mathrm{MBq})$.

Results: Six to 18 months after ablation, remission was observed in $60.0 \%$ of patients treated with low and in $60.0 \%$ of those treated with high RAI activities, biochemical disease was found in $18.8 \%$ of patients treated with low and in $14.3 \%$ of patients treated with high RAI activities, metastatic disease was found in $21.2 \%$ of patients treated with low and in $25.7 \%$ of patients treated with high RAI activities $(P=0.56)$. At the last follow-up (low activities, median 4.2 years; high activities, median 6.9 years), remission was observed in $76.5 \%$ of patients treated with low and in $72.1 \%$ of patients treated with high RAI activities, persistent disease was observed in $18.8 \%$ of patients treated with low and in $23.5 \%$ of patients treated with high RAI activities, recurrent disease was $2.4 \%$ in patients treated with low and $2.1 \%$ in patients treated with high RAI activities, deaths occurred in $2.4 \%$ of patients treated with low and in $2.1 \%$ of patients treated with high RAI activities $(P=0.87)$.

Conclusion: Our study provides the first evidence that in DTC patients at intermediate risk, high RAI activities at ablation have no major advantage over low activities.
\end{abstract}

European Journal of Endocrinology $16923-29$

\section{Introduction}

In patients with differentiated thyroid carcinoma (DTC), the rationale for post-surgical radioiodine ablation is to reduce the risk of recurrence and/or mortality and to facilitate patients' follow-up. According to the American Thyroid Association (ATA) and European Thyroid Association (ETA) guidelines $(1,2)$, sufficient evidences have been accumulated indicating that in low-risk patients who are candidates for thyroid ablation, low RAI activities between 1100 and $1850 \mathrm{MBq}$ have similar ablative effects $(3,4,5,6,7,8,9,10,11)$ and similar (very low) recurrence rates during follow-up $(12,13)$. Very recently, this concept has been reinforced by two prospective, randomized, multicenter studies $(14,15)$, which compared the effectiveness of RAI ablation using 1110 or $3700 \mathrm{MBq}$ of ${ }^{131} \mathrm{I}$. On the contrary, in high-risk patients, both guidelines $(1,2)$ recommend the use of high RAI activities (>3700 MBq). In between low- and high-risk patients, there is a group of patients defined at intermediate risk (microscopic invasion of tumor into the perithyroidal soft tissues, aggressive tumor histology, node positive) in whom no well-defined evidence can suggest the use of low or high RAI activities. Although based on expert opinions, the ATA guidelines (1) recommend activities between 3700 and $7400 \mathrm{MBq}$ of ${ }^{131}$ I. In addition, most series comparing the effectiveness of low or high RAI activities in patients at intermediate risk are focused on the rate of successful ablation but not on the impact that different activities of RAI may have on the long-term outcome of these patients. Our retrospective study was aimed to evaluate whether the use of low (1110-1850 MBq) or high $(\geq 3700 \mathrm{MBq}) \mathrm{RAI}$ activities for thyroid remnant ablation have an impact on the long-term outcome of patients who fulfilled the criteria of intermediate risk at the time of ablation. 


\section{Materials and methods}

In the database of DTC patients followed in our institution, we selected those $(n=225)$ who fulfilled the ATA criteria of intermediate risk: $\mathrm{i}$ ) any $\mathrm{T}$ any $\mathrm{N}$ with aggressive histology (tall cell, columnar, insular, and solid variant); ii) T3 with microscopic invasion of tumor into the perithyroidal soft tissues at initial surgery; and iii) any T-N1. Patients with distant metastases or positive AbTg were excluded from the study. All patients had been treated with near total thyroidectomy with $(n=86 / 225,38.2 \%)$ or without $(n=139 / 225,61.8 \%)$ lymph node dissection. Therapeutic lateral and centralcompartment lymph node dissection were performed in $46 / 86(53.5 \%)$ and $4 / 86(4.7 \%)$ patients respectively. Prophylactic lateral and central-compartment lymph node dissection were performed in 16/86 (18.6\%) and $20 / 86(23.3 \%)$ patients respectively. After surgery, all patients received RAI therapy at mean $( \pm$ s.D. $)$ activity of $3030 \pm 1213 \mathrm{MBq}$ (range 1110-7400 MBq), after thyroid hormone withdrawal (THW) $(n=110 / 225,48.9 \%)$ or after recombinant human TSH (rhTSH) administration $(n=115 / 225,51.1 \%)$.

At 6-18 months (median 9 months) after initial therapy, patients were evaluated by physical examination, neck ultrasound (US), and TSH-stimulated serum $\mathrm{Tg}$ measurement with or without diagnostic whole body scan (WBS) to define the clinical status. When stimulated serum $\mathrm{Tg}$ was undetectable $(<1.0 \mathrm{ng} / \mathrm{ml})$ and neck US (and diagnostic WBS when performed) were negative, patients were defined as in remission, and were shifted to annual follow-up based on serum $\mathrm{Tg}$ measurement on L-T $\mathrm{T}_{4}$ therapy and neck US. Additional TSH stimulation was performed in some cases during the follow-up. All patients with detectable basal and/or stimulated serum Tg were submitted to cross-sectional imaging (neck US, ${ }^{131}$ I WBS, ${ }^{18}$ FDG-PET, CT, MRI, and bone scan). Patients with detectable basal and/or stimulated serum $\mathrm{Tg}$ but no evidence of disease at imaging were classified as having biochemical disease. Patients with any evidence of disease at cross-sectional imaging were classified as having metastatic disease and were treated as appropriate. Recurrent disease was defined as the novel appearance of structural disease or biochemical disease (detectable $\mathrm{Tg}$ ) in patients previously classified in clinical remission.

\section{Diagnostic test}

Serum Tg, TSH, and TgAb were measured by chemiluminescent assay (Immulite 2000, DPC Diagnostic Products Corporation, Los Angeles, CA, USA). Tg assay had a functional sensitivity of $0.9 \mathrm{ng} / \mathrm{ml}$. A direct search for anti-Tg antibodies was performed using Immulite 2000 Anti-TG Ab (Siemens Medical Solutions Diagnostics, Los Angeles, CA, USA). It is a solidphase, enzyme-labeled, chemiluminescent sequential immunometric assay. Values below or equal to $20 \mathrm{IU} / \mathrm{ml}$ were regarded as negative.

Neck US was performed by experienced endocrinologists (members of our staff) using a high-resolution US color Doppler apparatus (AU 590 Asynchronous, Esaote Biomedica, Firenze, Italy) with a $7.5 \mathrm{MHz}$ linear transducer. Diagnostic or post-therapeutic ${ }^{131}$ I WBS was obtained using a one-head $\gamma$ camera (ApexSPX 4000; Elscint Italia, Milano, Italy) with a high-energy collimator and with a sensitivity of 160 c.p.m. $/ \mu \mathrm{Ci}$. The scan speed was $10 \mathrm{~cm} / \mathrm{min}$ with total counts of at least 100000 c.p.m.

\section{Statistical analysis}

Epidemiological data are presented as the mean \pm s.D., and with median when normality cannot be statistically established. The Anderson-Darling test was used to assess normality of variables' distribution. The $t$-test for independent data or the Mann-Whitney $U$ test was performed for normal or non-normal variables respectively. To evaluate significant differences in data frequency, we analyzed $2 \times 2$ contingency tables by the Fisher exact test. Tables with size larger than $2 \times 2$ were examined by $\chi^{2}$ test or a numerical approximation of the Fisher exact test, when all cell frequencies were $>4$ or not respectively. To test the difference between the proportion of adverse events in patients treated with low or high RAI activities and with rhTSH or $\mathrm{L}_{-} \mathrm{T}_{4}$ withdrawal (THW) at the time of the remnant ablation, we calculated the absolute risk reduction between the two groups of high and low RAI activities. Adverse outcome was evaluated at the end of a follow-up time with different subject-by-subject duration. We based it on a standard non-inferiority framework in which only absolute risk reduction $>20 \%$ was considered a clinically meaningful difference. For such analysis, the calculation of $95 \% \mathrm{CI}$ around the difference in outcome was used instead of a hypothesis test. To also take into account the follow-up time, a statistical survival analysis was also performed by comparing the Kaplan-Meier curves of the two groups of RAI activity and of the two different methods for thyroid remnant ablation (rhTSH or THW). As the favorable event of remission instead of the adverse event had to be considered in the analysis, remission curves instead of survival curves were compared. In the Kaplan-Maier analysis, only patients ever classified as in remission were included in the group of patients in remission whereas patients who had recurrent disease, persistent disease, or death were included in the group of patients with adverse outcome.

The equivalence between low and high RAI activities was tested using the Cox-Mantel log-rank test at a statistically significance level of $95 \%(P<0.05)$. Cox regression analysis was avoided because the too little sample size would give poor accuracy in model parameter estimates. Statistical analysis was performed using the StatView for Windows version 5.0.1 software (SAS Institute, Cary, NC, USA). 


\section{Results}

\section{Stratification of DTC patients and clinical characteristics}

As shown in Table 1, we stratified DTC patients in two groups, according to the RAI activity administered at the time of remnant ablation: $85 / 225$ patients $(37.8 \%)$ were treated with low RAI activities (1110-1850 MBq) and $140 / 225(62.2 \%)$ with high RAI activities $(\geq 3700 \mathrm{MBq})$. The two groups did not differ for age, histology, TNM, multifocality, diameter of primary tumor, lymph node dissection, type of lymph node metastasis, and basal/stimulated serum $\mathrm{Tg}$ at ablation. The follow-up was longer in the group of high RAI with respect to the group treated with low RAI activities (median 6.9 vs 4.2 years, $P<0.0001$ ).
Preparation for RAI ablation was more frequently performed with rhTSH when low activities were administered, reflecting the tendency to use rhTSH and low activities in recent years compared with patients treated before 2003. For the same reasons, the follow-up was longer in the group of high RAI with respect to the group treated with low RAI activities. The use of low or high RAI activities in patients with similar risk factors reflect the uncertainty of guidelines available up to now.

\section{Clinical outcome at the time of the first control (6-18 months after radioiodine remnant ablation) according to the RAI activities administered at ablation}

No difference in the time of the first follow-up $(P=0.68)$ was observed between patients treated with low or high

Table 1 Epidemiological and clinical features of the cohorts.

\begin{tabular}{|c|c|c|c|c|}
\hline & $\begin{array}{l}\text { Low RAl activities } \\
\qquad \mathrm{n}=85\end{array}$ & $\begin{array}{l}\text { High RAI activities } \\
\qquad n=140\end{array}$ & Test $^{a}$ & $\boldsymbol{P}$ \\
\hline \multicolumn{5}{|l|}{ Age } \\
\hline $\begin{array}{l}\text { Mean } \pm \text { S.D. } \\
\text { Range }\end{array}$ & $\begin{array}{c}42.8 \pm 16.9 \\
15-81\end{array}$ & $\begin{array}{c}45.2 \pm 16.3 \\
14-83\end{array}$ & $\mathrm{t}$ & 0.30 \\
\hline \multicolumn{5}{|l|}{ Gender } \\
\hline Male & $13(15.3 \%)$ & $46(32.9 \%)$ & $\mathrm{F}$ & 0.005 \\
\hline Female & $72(84.7 \%)$ & $94(67.1 \%)$ & & \\
\hline \multicolumn{5}{|l|}{ Histology } \\
\hline Papillary & $83(97.6 \%)$ & $130(92.9 \%)$ & $\mathrm{F}$ & 0.14 \\
\hline Follicular & $2(2.4 \%)$ & $10(7.1 \%)$ & & \\
\hline \multicolumn{5}{|l|}{ Aggressive variants } \\
\hline Yes & $18(21.2 \%)$ & $32(22.9 \%)$ & $\mathrm{F}$ & 0.87 \\
\hline No & $67(78.8 \%)$ & $108(77.1 \%)$ & & \\
\hline \multicolumn{5}{|l|}{ TNM } \\
\hline T1-T2N0-NX (aggressive histology) & $4(4.7 \%)$ & $7(5.0 \%)$ & $\chi^{2}$ & 0.34 \\
\hline T1-T2N1 & $15(17.6 \%)$ & $28(20.0 \%)$ & & \\
\hline T3NO-NX & $43(50.6 \%)$ & $54(38.6 \%)$ & & \\
\hline T3N1 & $23(27.1 \%)$ & $51(36.4 \%)$ & & \\
\hline \multicolumn{5}{|l|}{ Multifocality } \\
\hline Yes & 37 (43.5\%) & $56(40.0 \%)$ & $\mathrm{F}$ & 0.68 \\
\hline No & $48(56.5 \%)$ & $84(60.0 \%)$ & & \\
\hline \multicolumn{5}{|l|}{ Tumor diameter $(\mathrm{cm})$} \\
\hline Median, interquartile interval & $1.3,0.9-2.4$ & $1.5,1.0-3.0$ & MW & 0.14 \\
\hline Range & $0.3-6.5$ & $0.3-7.0$ & & \\
\hline \multicolumn{5}{|l|}{ Lymphadenectomy (central/lateral) } \\
\hline Yes & $30(35.3 \%)$ & $56(40.0 \%)$ & $\mathrm{F}$ & 0.57 \\
\hline No & $55(64.7 \%)$ & $84(60.0 \%)$ & & \\
\hline \multicolumn{5}{|l|}{ Lymphadenectomy (central/lateral) } \\
\hline Therapeutic & $20(66.7 \%)$ & $30(53.6 \%)$ & $\mathrm{F}$ & 0.26 \\
\hline Prophylactic & $10(33.3 \%)$ & $26(46.4 \%)$ & & \\
\hline \multicolumn{5}{|l|}{ Lymph node metastasis } \\
\hline Central (level VI) & $17(44.7 \%)$ & $41(51.9 \%)$ & $\mathrm{F}$ & 0.55 \\
\hline Later-cervical & $21(55.3 \%)$ & $38(48.1 \%)$ & & \\
\hline \multicolumn{5}{|l|}{ Preparation for radioiodine ablation } \\
\hline THW & $22(25.9 \%)$ & $88(62.9 \%)$ & $\mathrm{F}$ & $<0.0001$ \\
\hline rhTSH & $63(74.1 \%)$ & $52(37.1 \%)$ & & \\
\hline \multicolumn{5}{|l|}{$\mathrm{Tg}$ at ablation } \\
\hline Detectable (basal or stimulated) & $52(65.8 \%)$ & $79(72.5 \%)$ & $\mathrm{F}$ & 0.34 \\
\hline Undetectable (basal and stimulated) & $27(34.2 \%)$ & $30(27.5 \%)$ & & \\
\hline \multicolumn{5}{|l|}{ Follow-up duration (years) } \\
\hline Median, interquartile interval & $4.2,3.2-7.0$ & $6.9,5.2-8.9$ & MW & $<0.0001$ \\
\hline Range & $1.1-30.7$ & $1.1-30.8$ & & \\
\hline
\end{tabular}

${ }^{\mathrm{a}} t$, Student $t$, MW, Mann-Whitney; F, Fisher exact; $\chi^{2}$, Chi-squared. P values in bold indicate statistically significant difference. 
RAI activities (low activities, median 8.7 months, interquartile interval: 7.8-11.0; high activities median 9.1 months, interquartile interval: 7.7-12.4). At this time, no difference in clinical status was found between patients treated with low or high RAI activities (Fisher exact test, $P=0.56)$. In particular, $51 / 85(60.0 \%)$ patients treated with low and 84/140 (60.0\%) patients treated with high RAI activities fulfilled the criteria for remission. Biochemical disease (detectable serum $\mathrm{Tg}$ with no evidence of disease) was found in $16 / 85$ (18.8\%) patients treated with low and in 20/140 $(14.3 \%)$ patients treated with high RAI activities. Metastatic disease was found in 18/85 (21.2\%) patients treated with low and in 36/140 (25.7\%) patients treated with high RAI activities (Table 2).

Additional therapies were given to 76 patients and consisted of further courses of RAI activities (61.8\%), neck surgery $(9.2 \%)$, both surgery and radioiodine therapy $(13.2 \%)$, and a variety of other therapies, including tyrosine kinase inhibitors (15.8\%). The number of patients requiring additional therapies was not different (Fisher exact test, $P=0.11$ ) in patients treated with high $(53 / 140,37.9 \%)$ or low RAI activities $(23 / 85,27.1 \%)$.

\section{Clinical status at the last follow-up according to the RAI activities administered at ablation}

As shown in Table 1, at last follow-up (low activities, median 4.2 years; high activities, median 6.9 years, $P<0.0001)$, remission was observed in $65 / 85(76.5 \%)$ patients treated with low and in 101/140 (72.1\%) patients treated with high RAI activities, persistent disease (biochemical or metastatic) was observed in $16 / 85(18.8 \%)$ patients treated with low and in 33/140 (23.6\%) patients treated with high RAI activities, recurrence rate was observed in $2 / 85(2.4 \%)$ patients treated with low and in 3/140 $(2.1 \%)$ patients treated with high RAI activities, and deaths occurred in 2/85 (2.4\%) patients treated with low and in 3/140 (2.1\%) patients treated with high RAI activities. In no case were the differences statistically significant (Fisher exact test, $P=0.87$ ). When the same analysis was performed according to different features configuring patients at intermediate risk (T3NX-0, T1-2N1 or T1-2 with aggressive histology and T3N1), the results confirmed those obtained in all the series (Table 2). Both at the first control after initial therapy and at the final outcome, no difference was found according to low or high RAI activities in terms of remission, biochemical disease,

Table 2 Outcome after initial therapy and at the end of follow-up according to the RAI activities administered for remnant ablation in the entire group of patients and in the three subgroups defining the attribution to intermediate-risk class ( $P$ values refer to either Fisher exact, for $2 \times 2$ tables, or $\chi^{2}$ test, for tables of higher size).

\begin{tabular}{|c|c|c|c|c|}
\hline Variable & Outcome & Low RAl activities & High RAl activities & $\boldsymbol{P}$ \\
\hline \multicolumn{5}{|l|}{ All patients $(n=225)$} \\
\hline \multirow[t]{3}{*}{ 1st control after initial therapy } & Remission & $51(60.0 \%)$ & $84(60.0 \%)$ & \multirow[t]{3}{*}{0.56} \\
\hline & Biochemical disease & $16(18.8 \%)$ & $20(14.3 \%)$ & \\
\hline & Metastatic disease & $18(21.2 \%)$ & $36(25.7 \%)$ & \\
\hline \multirow[t]{3}{*}{ Final outcome } & Remission & $65(76.5 \%)$ & $101(72.1 \%)$ & \multirow[t]{3}{*}{0.87} \\
\hline & Recurrent disease & $2(2.4 \%)$ & $3(2.1 \%)$ & \\
\hline & Death & $2(2.4 \%)$ & $3(2.1 \%)$ & \\
\hline \multicolumn{5}{|l|}{ T3N0-X $(n=97)$} \\
\hline \multirow[t]{3}{*}{ 1st control after initial therapy } & Remission & $32(74.4 \%)$ & $39(72.2 \%)$ & \multirow[t]{3}{*}{0.67} \\
\hline & Biochemical disease & $7(16.3 \%)$ & $7(13.0 \%)$ & \\
\hline & Metastatic disease & $4(9.3 \%)$ & $8(14.8 \%)$ & \\
\hline \multirow[t]{3}{*}{ Final outcome } & Remission & $34(79.1 \%)$ & $43(79.6 \%)$ & \multirow[t]{3}{*}{0.86} \\
\hline & Recurrent disease & $2(4.7 \%)$ & $1(1.9 \%)$ & \\
\hline & Persistent disease & $6(14.0 \%)$ & $9(16.7 \%)$ & \\
\hline \multicolumn{5}{|c|}{ T1-2N1 and T1-2N0 with aggressive histology $(n=54)$} \\
\hline \multirow[t]{3}{*}{ 1st control after initial therapy } & Remission & $10(52.6 \%)$ & $21(60.0 \%)$ & \multirow[t]{3}{*}{0.84} \\
\hline & Biochemical disease & $5(26.3 \%)$ & $7(20.0 \%)$ & \\
\hline & Metastatic disease & $4(21.1 \%)$ & $7(20.0 \%)$ & \\
\hline \multirow[t]{3}{*}{ Final outcome } & Remission & $15(78.9 \%)$ & $26(74.3 \%)$ & \multirow[t]{3}{*}{0.74} \\
\hline & Recurrent disease & $0(0 \%)$ & $1(2.9 \%)$ & \\
\hline & Persistent disease & $4(21.1 \%)$ & $8(22.9 \%)$ & \\
\hline \multicolumn{5}{|l|}{ T3N1 $(n=74)$} \\
\hline \multirow[t]{3}{*}{ 1st control after initial therapy } & Remission & $9(39.1 \%)$ & $24(47.1 \%)$ & \multirow[t]{3}{*}{0.73} \\
\hline & Biochemical disease & $4(17.4 \%)$ & $6(11.8 \%)$ & \\
\hline & Metastatic disease & $10(43.5 \%)$ & $21(41.2 \%)$ & \\
\hline \multirow[t]{4}{*}{ Final outcome } & Remission & $16(69.6 \%)$ & $32(62.7 \%)$ & \multirow[t]{4}{*}{0.86} \\
\hline & Recurrent disease & $0(0 \%)$ & $1(2.0 \%)$ & \\
\hline & Persistent disease & $6(26.1 \%)$ & $16(31.4 \%)$ & \\
\hline & Death & $1(4.3 \%)$ & $2(3.9 \%)$ & \\
\hline
\end{tabular}


Table 3 Clinical outcome at the end of follow-up according to the RAl activity administered (comparison 1) and method of preparation (comparison 2) at the time of remnant ablation.

\begin{tabular}{|c|c|c|c|c|}
\hline \multirow[b]{2}{*}{ Variable } & \multicolumn{2}{|c|}{ Comparison 1} & \multicolumn{2}{|c|}{ Comparison 2} \\
\hline & Low RAI activities & High RAI activities & rhTSH preparation & THW preparation \\
\hline $\begin{array}{l}\text { Clinical remission at the last follow-up } \\
\text { Absolute risk reduction }{ }^{\mathrm{a}}(95 \% \mathrm{Cl})\end{array}$ & \multicolumn{2}{|c|}{$0.043(-0.067 \infty)$} & $\begin{array}{r}90 / 115(78.3 \%) \\
0.092(-\end{array}$ & $\begin{array}{l}76 / 110(69.1 \%) \\
014 \infty)\end{array}$ \\
\hline
\end{tabular}

${ }^{\mathrm{a} B y}$ non-inferiority test.

metastatic disease, recurrence, or death in the three different categories of patients.

In addition, as shown in Table 3, the non-inferiority test supplies a value of absolute risk reduction of 0.043 , with a lower bound of $95 \%$ CI equal to - 0.067 , between patients treated with low and with high RAI activities. The CI had a lower bound greater than the selected level of $-20 \%$, proving the non-inferiority of low RAI activity.

\section{Relationship between remission probability and follow-up time in low and high RAI activities}

Figure 1 shows the Kaplan-Meier curves for evaluating the remission probability in the two groups of RAI activities. Curve comparison to test the equivalence of remission behavior, as a function of follow-up time, gave no statistical significance $(P=0.38)$. This confirms that low and high RAI activities does not differently influence the remission probability.

When the same analysis was performed according to different features configuring patients at intermediate risk (T3NX-0, T1-2N1, or T1-2 with aggressive histology and T3N1), the results confirmed those obtained in all the series (Fig. 1).

\section{Absolute risk reduction and relationship between remission probability and follow-up time according to the methods of preparation (rhTSH or THW)}

Preparation by rhTSH or THW had no influence on the final outcome in both the groups treated with high or low RAI activities (rhTSH, median follow-up 4.5 years; THW, median follow-up 8.4 years, $P<0.0001$ ). As shown in Table 3, the non-inferiority test supplies a value of absolute risk reduction of 0.092 (95\% CI lower bound $=-0.014$ ) between patients prepared for radioiodine thyroid remnant ablation with rhTSH or THW. The CI had a lower bound greater than the selected level of $-20 \%$, proving the non-inferiority of preparation with rhTSH. Figure 2 shows the Kaplan-Meier curves for evaluating the remission probability in the two groups (rhTSH and THW) according to the RAI activities administered. Curve comparison to test the
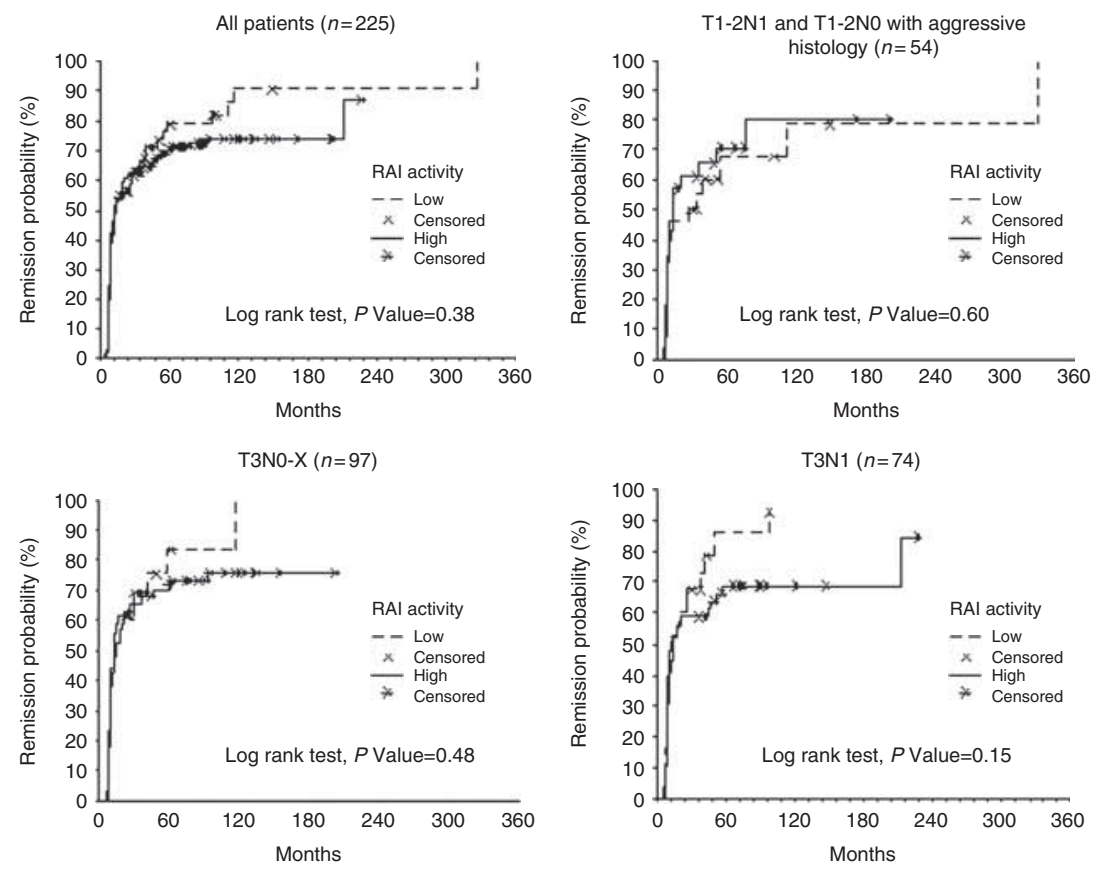

Figure 1 Kaplan-Meier curves of remission probability for low and high RAl activities in all DTC patients $(n=225)$ and in the three subgroups defined according to different features configuring DTC patients at intermediate risk: T1-2N1 and T1-2N0 with aggressive histology $(n=54)$ and T3NX-0 $(n=97)$ and T3N1 $(n=74)$. 
$\operatorname{THW}(n=100)$

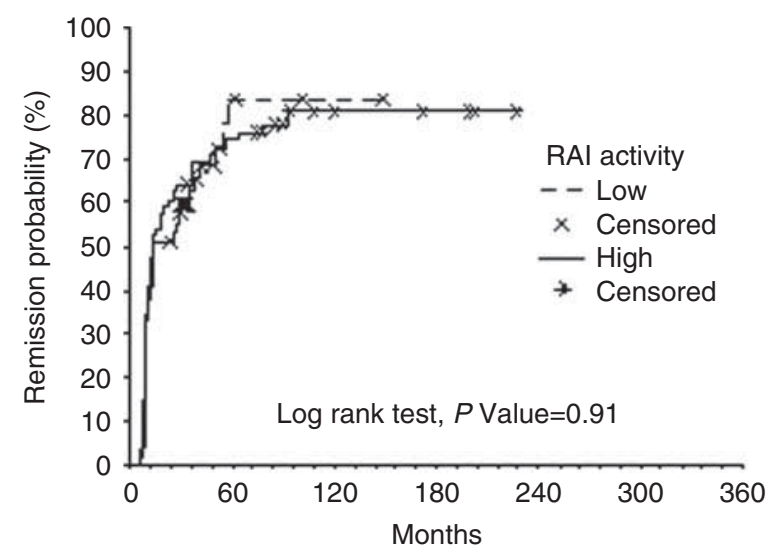

$\operatorname{rhTSH}(n=115)$

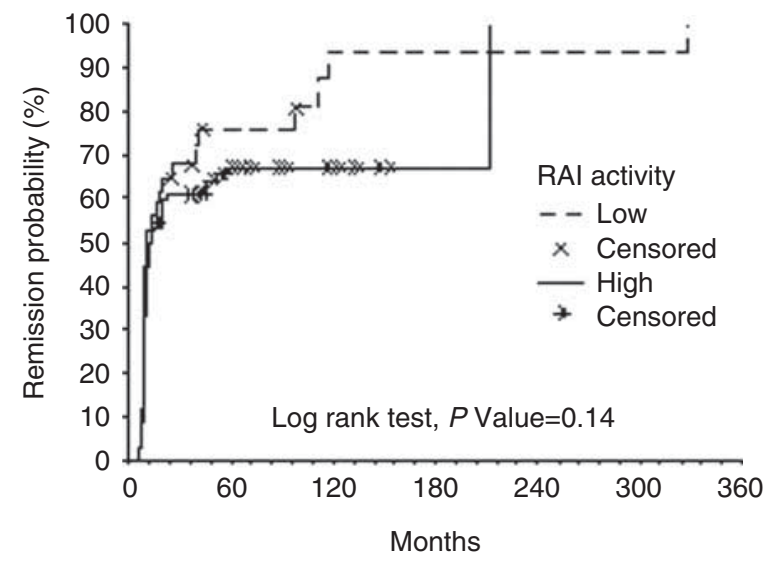

Figure 2 Kaplan-Meier curves of remission probability for low and high RAI activities in DTC patients prepared for remnant ablation after THW $(n=110)$ or prepared after rhTSH $(n=115)$.

equivalence of remission behavior, as function of followup time, gave no statistical significance in both groups (rhTSH group, $P=0.14$ and THW group, $P=0.91$ ).

\section{Discussion}

In patients with DTC, radioiodine ablation aims to reduce the risk of recurrence and/or mortality and to facilitate patients' follow-up. In low-risk patients, several series, including two recent prospective, multicenter studies $(3,4,5,6,7,8,9,10,11,12,13,14$, 15), have compared the effectiveness of RAI ablation when using low RAI activities (1110-1850 MBq) or high RAI activities ( $\geq 3700 \mathrm{MBq}$ ) and, in general, their results indicate that low RAI activities are as effective as high RAI activities. In patients defined as high risk according to ATA and ETA guidelines $(1,2)$, high RAI activities are recommended. Much debate still exists regarding the activities administered in intermediate risk (microscopic invasion of tumor into the perithyroidal soft tissues, aggressive tumor histology, node positive). In this context, no evidences are provided regarding the optimal RAI activity to be administered and current recommendations, advocating the use of high RAI activities, are based mainly on the expert opinion rather than clinical evidence $(1,2)$.

The novelty of our study was to evaluate, retrospectively, the final outcome (at a mean follow-up of 7 years) of patients ablated with low (1110-1850 MBq) or high RAI activities $(\geq 3700 \mathrm{MBq})$, who were considered at intermediate risk, according to current guidelines $(1,2)$. Our study shows that in these patients, low or high RAI activities result in similar outcomes both in terms of remission, recurrences, persistent disease, and deaths. Our results are in agreement with a previous report by Mazzaferri (16), who compared the outcome of 100 patients stage II-III (to some extent comparable with intermediate-risk patients) treated with low (1110-1850 MBq) or high (>1850-7400 MBq) RAI activities. After a mean follow-up of 15 years, the recurrence rate was $7 \%$ in patients treated with low RAI activities and 9\% in those treated with high activities. Another evidence of our study is that the RAI activities administered had similar outcomes even when analyzing the data according to the method of preparation for RAI ablation (THW or rhTSH), in agreement with other studies in low- or high-risk patients $(17,18,19)$. However, in these studies, the authors used only high RAI activities.

As for other retrospective studies, our results have the potential bias of the RAI activities selected in different patients. Indeed, the preparation for RAI ablation was more frequently performed after THW and using high RAI activities, but this occurred mainly in patients treated in the past years. In most recent years, we shifted to the use of rhTSH in most cases, using low RAI activities. As a consequence, the other possible bias is that patients treated with high RAI activities have longer follow-up (median 6.9 years) compared with patients treated with low RAI activities (median 4.2 years) as well as patients prepared by THW have longer follow-up (median 8.4 years) compared with patients prepared by rhTSH (median 4.5 years). This possible bias is ruled out by the Kaplan-Meier analysis of remission probability (Figs 1 and 2). In addition, to further evaluate the possible bias due to the different length of follow-up, we compared the proportion of patients ever considered in remission between the groups (low vs high RAI activities, rhTSH vs THW, data not shown). We observed that the remission rate was slightly greater in patients treated with low compared with those treated with high RAI activities (76.5 vs $72.1 \%$ respectively) and in patients prepared by rhTSH compared with those prepared by THW (78.3 vs $69.1 \%$ respectively). These results might suggest that the remission rate in patients treated with low activities or prepared by rhTSH would not be significantly lower 
compared with that observed in patients treated with high RAI activity or prepared by THW, even with a longer follow-up.

In conclusion, our study, despite the above-mentioned limitations, provide a first evidence that in DTC patients classified at intermediate risk, the RAI activity (low or high) or methods of preparation (THW or rhTSH) used for ablation has no impact on the subsequent outcome. Similar to current practice in low-risk patients, intermediate-risk patients may also be safely ablated using low RAI activity after rhTSH preparation.

\section{Declaration of interest}

The authors declare that there is no conflict of interest that could be perceived as prejudicing the impartiality of the research reported.

\section{Funding}

This work was supported in part by grants from Ministero Italiano dell'Università e Ricerca (MIUR, 2008) (grant number 200845 P777_001).

\section{References}

1 Cooper DS, Doherty GM, Haugen BR, Kloos RT, Lee SL, Mandel SJ, Mazzaferri EL, McIver B, Pacini F, Schlumberger M et al. Revised American Thyroid Association management guidelines for patients with thyroid nodules and differentiated thyroid cancer. Thyroid 200919 1167-1214. (doi:10.1089/thy.2009.0110)

2 Pacini F, Schlumberger M, Dralle H, Elisei R, Smit JW, Wiersinga W \& European Thyroid Cancer Taskforce. European consensus for the management of patients with differentiated thyroid carcinoma of the follicular epithelium. European Journal of Endocrinology 2006 154 787-803. (doi:10.1530/eje.1.02158)

3 Creutzig H. High or low dose radioiodine ablation of thyroid remnants? European Journal of Nuclear Medicine 198712 500-502. (doi:10.1007/BF00620474)

4 Johansen K, Woodhouse NJY \& Odugbesan O. Comparison of $1073 \mathrm{MBq}$ and $3700 \mathrm{MBq}$ iodine-131 in post-operative ablation of residual thyroid tissue in patients with differentiated thyroid cancer. Journal of Nuclear Medicine 199132 252-254.

5 Bal CS, Padhy AK, Jana S, Pant GS \& Basu AK. Prospective randomized clinical trial to evaluate the optimal dose of ${ }^{131} \mathrm{I}$ for remnant ablation in patients with differentiated thyroid carcinoma. Cancer 199677 2574-2580. (doi:10.1002/(SICI)10970142(19960615)77:12<2574::AID-CNCR22> 3.0.CO;2-O)

6 Bal CS, Kumar A \& Pant GS. Radioiodine dose for remnant ablation in differentiated thyroid carcinoma: a randomized clinical trial in 509 patients. Journal of Clinical Endocrinology and Metabolism 2004 89 1666-1673. (doi:10.1210/jc.2003-031152)

7 Bal C, Chandra P, Kumar A \& Dwivedi S. A randomized equivalence trial to determine the optimum dose of iodine-131 for remnant ablation in differentiated thyroid cancer. Nuclear Medicine Communications 201233 1039-1047. (doi:10.1097/ MNM.Ob013e32835674af)

8 Caglar M, Bozkurt FM, Akca CK, Vargol SE, Bayraktar M, Ugur O \& Karaağaoğlu E. Comparison of 800 and $3700 \mathrm{MBq}$ iodine-131 for the postoperative ablation of thyroid remnant in patients with lowrisk differentiated thyroid cancer. Nuclear Medicine Communications 201233 268-274. (doi:10.1097/MNM.0b013e32834ec5d6)
9 Fish SA, Basu S, Alavi A \& Mandel SJ. Comparison of efficacy of $2220 \mathrm{MBq}$ versus $3700 \mathrm{MBq} \mathrm{I}-131$ for ablation of thyroid remnant in patients with differentiated thyroid cancer. Quarterly Journal of Nuclear Medicine and Molecular Imaging $2010 \mathbf{5 4}$ $560-563$.

10 Pilli T, Brianzoni E, Capoccetti F, Castagna MG, Fattori S, Poggiu A, Rossi G, Ferretti F, Guarino E, Burroni L et al. A comparison of $1850(50 \mathrm{mCi})$ and $3700 \mathrm{MBq}(100 \mathrm{mCi})$ 131-iodine administered doses for recombinant thyrotropin-stimulated postoperative thyroid remnant ablation in differentiated thyroid cancer. Journal of Clinical Endocrinology and Metabolism 200792 3542-3546. (doi:10.1210/jc.2007-0225)

11 Chianelli M, Todino V, Graziano FM, Panunzi C, Pace D, Guglielmi R, Signore A \& Papini E. Low-activity $(2.0 \mathrm{GBq}$; $54 \mathrm{mCi}$ ) radioiodine post-surgical remnant ablation in thyroid cancer: comparison between hormone withdrawal and use of rhTSH in low-risk patients. European Journal of Endocrinology 2009 160 431-436. (doi:10.1530/EJE-08-0669)

12 Kukulska A, Krajewska J, Gawkowska-Suwińska M, Puch Z, Paliczka-Cieslik E, Roskosz J, Handkiewicz-Junak D, Jarzab M, Gubała E \& Jarzab B. Radioiodine thyroid remnant ablation in patients with differentiated thyroid carcinoma (DTC): prospective comparison of long-term outcomes of treatment with 30, 60 and $100 \mathrm{mCi}$. Thyroid Research 20103 9. (doi:10.1186/17566614-3-9)

13 Schvartz C, Bonnetain F, Dabakuyo S, Gauthier M, Cueff A, Fieffé S, Pochart JM, Cochet I, Crevisy E, Dalac A et al. Impact on overall survival of radioactive iodine in low-risk differentiated thyroid cancer patients. Journal of Clinical Endocrinology and Metabolism 201297 1526-1535. (doi:10.1210/jc.2011-2512)

14 Mallick U, Harmer C, Yap B, Wadsley J, Clarke S, Moss L, Nicol A, Clark PM, Farnell K, McCready R et al. Ablation with low-dose radioiodine and thyrotropin alfa in thyroid cancer. New England Journal of Medicine 2012366 1674-1685. (doi:10.1056/ NEJMoa1109589)

15 Schlumberger M, Catargi B, Borget I, Deandreis D, Zerdoud S, Bridji B, Bardet S, Leenhardt L, Bastie D, Schvartz C et al. Strategies of radioiodine ablation in patients with low-risk thyroid cancer. New England Journal of Medicine $2012 \quad 366 \quad 1663-1673$. (doi:10.1056/NEJMoa1108586)

16 Mazzaferri EL. Thyroid remnant ${ }^{131} \mathrm{I}$ ablation for papillary and follicular thyroid carcinoma. Thyroid $1997 \mathbf{7}$ 265-271. (doi:10.1089/thy.1997.7.265)

17 Hugo J, Robenshtok E, Grewal R, Larson SM \& Tuttle RM Md. Recombinant human TSH-assisted radioactive iodine remnant ablation in thyroid cancer patients at intermediate to high risk of recurrence. Thyroid 201222 1007-1015. (doi:10.1089/thy. 2012.0183)

18 Tuttle RM, Brokhin M, Omry G, Martorella AJ, Larson SM, Grewal RK, Fleisher M \& Robbins RJ. Recombinant human TSHassisted radioactive iodine remnant ablation achieves short-term clinical recurrence rates similar to those of traditional thyroid hormone withdrawal. Journal of Nuclear Medicine $2008 \mathbf{4 9}$ 764-770. (doi:10.2967/jnumed.107.049072)

19 Elisei R, Schlumberger M, Driedger A, Reiners C, Kloos RT, Sherman SI, Haugen B, Corone C, Molinaro E, Grasso L et al. Follow-up of low-risk differentiated thyroid cancer patients who underwent radioiodine ablation of postsurgical thyroid remnants after either recombinant human thyrotropin or thyroid hormone withdrawal. Journal of Clinical Endocrinology and Metabolism 2009 94 4171-4179. (doi:10.1210/jc.2009-0869)

Received 31 October 2012

Revised version received 19 March 2013

Accepted 17 April 2013 